Schweiz. Z. Tuberk. 1954;11:I-IV

\title{
Contents, Vol. 11, 1954
}

\section{INDEX}

Amsler, H., vide Kaufmann, G.

Austrian, H., vide Moeschlin, S.

Barazzone, J., vide Fabre, J.

Berlinger, R., Zur Diagnose und Therapie der Spondylitis tuberculosa vor und nach Einführung der Tuberkulostatica 467

Böhm, F., Beitrag zur Genese des echten Tuberkulom $\beta \quad 416$

Bonard, E. C, Tuberculose abdominale et phtisie pulmonaire

Bucher, K. und U. Lanz, Änderungen des Lungenvolumens durch Pneumothorax 146

Chadourne et Pinellí, Discussion 286

Chenet, $\mathrm{C}$, vide Curchod, B.

Curchod, B., C. Chenet et S. Vielhauer, Effet retard de la Butazolidine sur

l'élimination du PAS 176

Dölker, B., Segmentdiagnostik bei Lungentuberkulose und ihre Beziehungen

zur Kollapsindikation 47

Eerland, L. D. und K. K. M. F. Seghers, Segmentresektion bei Lungentuber

kulose 353

Eidus, L., vide Lãnyí, M.

Fabre, J. et J. Barazzone, Les alterations du myocarde dans la tuberculose pulmonaire 129

Favez, G., Comport ement et usage thérapeutique des acides amines chez les tuberculeux pulmonaires

97

Froehlích, IV., Anatomisch-pathologischer Beitrag der oralen Blutungen

bei Lungenerkrankungen 290

Galy, P., L'influence de la structure segmentaire du poumon sur revolution

de la tuberculose 264

Haeflíger, E. und G. Mark, Die Bedeutung der Lungensegmente für die kli-

nische Pathologie der Tuberkulose 247

Hartl, W., Erste Erfahrungen mit Reazide bei experimenteller Tuberkulose 65 - Experimenteller

Beitrag zur Behandlung der Lungentuberkulose mit para-

Aminobenzoesäure 369

Hírsch, A., Zur Frage der Häufigkeit von Komplikationen bei chirurgischer

Kollapstherapie nach intrakavitärer Injektionsbehandlung 210

Hoess, if., Zur medikamentösen Behandlung der Nierentuberkulose .... 329 Huber, K., Die

Bedeutung der tuberkulösen Hiluslymphknotenperforation in

der Differentialdiagnose akuter fieberhafter Erkrankungen 439

Hug, H., Klinik und Pathogenese des Bronchialadenoms 36

Kanzler, W., Die behandelte Nierentuberkulose im Röntgenbild 
Kaufmann, G., J. Wanner und H. Amsler, Tuberkelbazillen in resezierten Lungenherden 322

Kehler, E., Endrokrines System und Tuberkulose im Rahmen der vegetativen Regulationen 185

Küchler, W., tlber ein neues kombiniertes, perorales Tuberkuloseheilmittel . 344

Künzler, R., Ein Fall von Hämangioendotheliom der Lunge 401

Lãnyi, M. und L. Eidus, Über die biologische Desintegration der Tuberkel bazillen $\quad 447$

Lanz, U., vide Bucher, K.

Maassen, W., Die ein- und doppelseitige Bronchographie in Endotrachealnarkose bei offener Lungentuberkulo.se 427

Malluche,.//.. Der extrapleurale Pneumothorax als Abschluß nach Kavernendrainage 113

Mark, G., vide Haefliger, E.

Moeschlín, S. and if. Austrian, The Effect of a Subtherapeutic Dose of Strep tomycin combined with a Low Dose of Isoniazid on Experimental Tuber culosis in Guinea-Pigs 56

Morin, J., «Marsilid» 296

Naef, A. P., Les succès de l'exérèse pour tuberculose pulmonaire dans les cas désespérés, appareminent inopérables 308

- Schlußwort-Remarque finale 321

Nissen, R., Diskussion $\quad 320$

Ramer, Z., vide Wehrlin, $\mathrm{H}$.

Scheu, H., Vorläufige Mitteilung über Beobachtungen bei Behandlung der Lungeatuberkulose mil dem Hydrazid der Cyanessigsäure (Reazide) . 77

Schneider, S., Période tardive et abandon de la pneumolyse extrapleurale . . 378 Seghers, K. K. M. F., vide Eerland, L. D.

Steínlín, H., Die Kombinationsbehandlung der kavernösen Lungentuberkulose mit INH und intravenösen PAS-Infusionen 302

Stephani, J., Passage d'une maladie de Besnier-Boeck bénigne du poumon, à une tuberculose ulcereuse à marche torpide 201

Töndury, G., Zur Segment-Anatomic der Lungenlappen 227

Uehlínger, E., Lungensegment und Lungentuberkulo.se 237

Undritz, E., Diskussion 288

Vielhauer, S., vide Curchod, B.

Wanner, J., vide Kaufmann, G.

Wehrlin, H. und Z. Ramer, Die Bedeutung der Tomographie für Diagnose und Beurteilung der Knochen- und Gelenkstuberkulose 161

Wernsdorfer, G., Untersuchungen über den Einfluß der Exposition auf die Tuberkulinreaktivität und die Morbidität bei Personen aus der Umgebung $\tau$ on Tuberkulösen $\quad 455$

Zuídeman, P., Diskussion 286

GESELLSCHAFTSBERICHTE SOCIÉTES - SOCIETY TRANSACTIONS

Schweizerische Vereinigung gegen die Tuberkulose/Gesellschaft Schweize-

rischer Tuberkuloseärzte: 34.wissenschaftliche Tagung in Basel, 3./4. April 1954. Association Suisse centre la Tuberculose/Société des 
Médecins Suisses specialises en Tuberculose: $34 \mathrm{e}$ reunion scientifique à Bale, les 3 et 4 avril $1954 \quad 225$

Corrigendum 160

Kongresse - Reunions - Meetings

159

Libri 92, 153, 221, 348, 420, 495

Varia 424 